121 Abs. 2 AEUV und die beschäftigungspolitischen Leitlinien gem. Art. 148 AEUV beschlossen. Diese durch die vorgenannten Rechtsinstrumente umgesetzten Leitlinien bilden zusammen die integrierten Leitlinien für die Umsetzung der Strategie Europa 2020. In den folgenden 10 integrierten Leitlinien zu Europa 2020 wird der Rahmen für die Strategie Europa 2020 sowie für Reformen auf der Ebene der Mitgliedstaaten abgesteckt:

1. Gewährleistung der Qualität und langfristigen Tragfähigkeit der öffentlichen Finanzen,

2. Beseitigung makroökonomischer Ungleichgewichte,

3. Abbau von Ungleichgewichten in der Eurozone,

4. Optimierung der FuE- sowie der Innovationsförderung, Stärkung des Wissensdreiecks und Freisetzung des Potenzials der digitalen Wirtschaft,
5. Verbesserung der Ressourceneffizienz und Abbau der Treibhausgasemissionen,

6. Verbesserung der Rahmenbedingungen für Unternehmen und Verbraucher und Modernisierung der industriellen Basis,

7. Erhöhung der Beschäftigungsquote und Abbau der strukturellen Arbeitslosigkeit,

8. Heranbildung von Arbeitskräften, deren Qualifikationen den Anforderungen des Arbeitsmarkts entsprechen, Förderung der Arbeitsplatzqualität und des lebens-langen Lernens,

9. Steigerung der Leistungsfähigkeit der allgemeinen und beruflichen, Bildungssysteme auf allen Ebenen und Verbesserung des Zugangs zur Hochschulbildung,

10.Bekämpfung von gesellschaftlicher Ausgrenzung und Armut.

\title{
„eg-check geprüft!“ - Ergebnisse einer betrieblichen Anwendung
}

\section{Sarah Lillemeier}

Doktorandin bei der Hans Böckler Stiftung - WSI, Düsseldorf

Der Entgeltgleichheitsgrundsatz ist rechtlich in Deutschland bereits seit über einem halbem Jahrhundert verankert. Dennoch wird die Einhaltung dieses Grundsatzes nicht kontrolliert sondern den Unternehmen selbst überlassen. Wie diese das sehr komplexe Feld der Entgeltungleichheit systematisch angehen sollen, blieb in der Vergangenheit häufig unklar. Inzwischen existieren allerdings Instrumente, mit denen das Thema auf der betrieblichen Ebene bearbeitet werden kann.

Der eg-check ist ein solches Instrumentarium, mit dem Ungleichbehandlungen der Geschlechter beim Arbeitsentgelt sichtbar gemacht, konkrete Ursachen aufgezeigt und das finanzielle Ausmaß einer Benachteiligung berechnet werden können. Im Gegensatz zu anderen bestehenden Instrumenten basiert er auf dem geltenden Recht in Deutschland. D.h. mit dem eg-check können Entgeltdiskriminierungen aufgrund des Geschlechts aufgedeckt und beseitigt werden. Entwickelt wurde das Instrumentarium im Jahr 2010 von Karin Tondorf und Andrea Jochmann-Döll.

Ausgehend von dem Interesse und der Bereitschaft eines großen Versorgungsunternehmens die eigenen Gehaltsstrukturen auf Ungleichbehandlungen zu überprüfen, ist der eg-check zur praktischen Anwendung gekommen. Aus den vielfältigen Prüfmöglichkeiten die mit diesem Instrumentarium möglich sind, wurde eine konkrete Prüffrage ausgewählt. „Wird Frauen und Männern für gleichwertige Arbeit ein gleiches Grundentgelt gezahlt? “ Die Entscheidung für diese partielle Prüfung ist gefallen, da das Grundentgelt einen Großteil des Verdienstes ausmacht. Darüber hinaus kann mit dieser Frage die vorgenommene Arbeitsbewertung im Unternehmen kri- tisch betrachtet werden. Diese ist grundsätzlich von Interesse, indem darin eine wesentliche Ursache für Entgeltdiskriminierungen aufgrund des Geschlechts begründet liegen kann.

Zur Beantwortung der ausgewählten Prüffrage wurden insgesamt zwei Instrumente aus dem eg-check verwendet. Der Regelungscheck beinhaltet zur Prüfung der Grundentgeltfindung 14 Fragen, die an die schriftlich niedergelegten Regelungen zur Arbeitsbewertung gestellt werden. Anhand dieses Fragenkatalogs lassen sich Regelungen identifizieren, die ein Diskriminierungspotential in sich bergen. Neben den kritischen Regelungen können Benachteiligungen aber auch durch die praktische Anwendung entstehen. Aus diesem Grund wurden zudem ausgewählte Paarvergleiche aus dem eg-check durchgeführt. Mit den Paarvergleichen lassen sich die betrieblichen Eingruppierungen auf Diskriminierungen hin überprüfen.

Für das geprüfte Unternehmen gilt ein Tarifvertrag. Darin festgelegt ist das Verfahren der Arbeitsbewertung, dass jeweils auf der betrieblichen Ebene angewendet wird. Der Regelungscheck hat ergeben, dass in diesem Verfahren insgesamt sechs kritische Regelungen mit Diskriminierungspotentialen enthalten sind:

1. Die Kriterien die im Tarifvertrag zur Bewertung der Tätigkeiten zur Verfügung stehen, sind unvollständig und zwar insofern, als dass sie nicht die charakteristischen Anforderungen und Belastungen der Tätigkeiten von männlichen und weiblichen Beschäftigten abbilden. Der Europäische Gerichtshof (EuGH) hat mit seiner Rechtssprechung festgelegt, dass die verwendeten Kriterien das „Wesen“ der Arbeit widerspiegeln sollen und auch solche Kriterien zu berücksichtigen sind „hinsichtlich derer die weiblichen Arbeitnehmer besonders geeignet sein können " (EuGH v. 1.7.1986 Rs. C - 237/85 „Rummler“). Im Tarifvertrag 


\section{Antidiskriminierungsstelle des Bundes fördert eg-check-Projekte}

„Es liegt im ureigenen Interesse von Unternehmen und öffentlichen Verwaltungen, dass Frauen in der Entlohnung nicht benachteiligt werden“, sagt Christine Lüders, Leiterin der Antidiskriminierungsstelle des Bundes. Daher führt sie gemeinsam mit engagierten Unternehmen aus dem privaten und öffentlichen Sektor eine Analyse der Entgeltgleichheit mit dem Instrument eg-check. de durch. Die beteiligten Unternehmen werden öffentlichkeitswirksam begleitet und für ihr Engagement ausgezeichnet.

stehen einzig und allein die Qualifikationen und die Übernahme von Verantwortung zur Bewertung zur Verfügung. Unberücksichtigt bleiben hingen Belastungen, sowohl psycho-sozialer als auch physischer Art. Insbesondere die psycho-soziale Anforderungen sind aber häufig relevant bei frauendominierten Tätigkeiten. Indem dieses Kriterium im Bewertungssystem fehlt, können diese Aspekte jedoch nicht berücksichtigt und damit auch nicht entlohnt werden. Die physischen Anforderungen, die häufig im Zusammenhang männlichdominierter Tätigkeiten auftreten, sind zwar im Verfahren der Arbeitsbewertung nicht vorgesehen. Sie können aber über Zuschlagsregelungen (Erschwerniszuschläge) miteinbezogen werden. In diesem Vorgehen kann jedoch ebenfalls ein erhebliches Diskriminierungspotential begründet sein, welches allerdings eigenständig zu prüfen wäre. Im eg-check sind die notwendigen Instrumente enthalten, um auch Zuschlagsregelungen zu prüfen.

2. Die im Tarifvertrag benannten Bewertungskriterien und ihre Differenzierungsmerkmale sind nicht eindeutig umschrieben. Z.B. ist nicht festgelegt, was unter „Verantwortung“ zu verstehen ist, wie „besondere Spezialkenntnisse“ definiert sind oder wann für eine Tätigkeit das Merkmal „besondere Schwierigkeit und Bedeutung“ erfüllt ist. Aufgrund dieser fehlenden Eindeutigkeiten, müssen die Kriterien von den bewertenden Personen auf der betrieblichen Ebene ausgelegt werden. Diese Interpretationsspielräume wiederum können Diskriminierungen begründen, indem sie zuungunsten eines Geschlechts ausgelegt werden.

3. An einer Stelle im Tarifvertrag werden zwei Kriterien aneinandergereiht, um eine bestimmte Entgeltgruppe zu erreichen. Dadurch können „selbstständige Leistungen“ nur bewertet werden, wenn die Ausübung der Tätigkeit zusätzlich „gründliche und vielseitige Fachkenntnisse“ erfordert. Ein solches Vorgehen bedingt jedoch, dass „selbständige Leistungen" nicht separat bewertet werden können. Als Konsequenz könnten die möglichen „selbstständigen Leistungen“ im Rahmen von frauendominierten Tätigkeiten, nicht bewertet werden, wenn gleichzeitig beispielsweise nur „gründliche Fachkenntnisse “ erforderlich sind. Hierin liegt ein wesentliches Diskriminierungspotential begründet.

4. Die Grundlage für eine diskriminierungsfreie Arbeitsbewertung ist eine geschlechtsneutrale Arbeitsbeschreibung. Denn prinzipiell gilt, was nicht beschrieben ist, kann auch nicht bewertet und damit auch nicht entlohnt werden. Im Tarifvertrag ist eine solche Arbeitsbeschreibung nicht festgeschrieben, was dazu führen kann, dass auf der betrieblichen Ebene Arbeitsbewertungen auch ohne fundierte Grundlage vorgenommen werden. Ein solches Vorgehen vergrößert jedoch den ohnehin gegebenen Interpretationsspielraum und die damit verbundene Anfälligkeit für Diskriminierungen.

5. Im Tarifvertrag wird ein summarisches Arbeitsbewertungssystem verwendet. Die innere Logik dieses Verfahrens bedingt, dass die einzelnen Kriterien, die zur Bewertung zur Verfügung stehen, pauschal bewertet werden. Dadurch ist nicht erkennbar, welches Gewicht den einzelnen Kriterien zukommt. Das Verfahren bietet somit mehr Auslegungsspielraum und ist weniger durchschaubar und nachvollziehbar. Auch an diesen Stellen können Benachteiligungen aufgrund des Geschlechts begründet liegen.

6. Das Entgeltgefüge des Tarifvertrag sieht vor, dass in den höhern Entgeltgruppen die zugeordneten Beträge überdurchschnittlich im Vergleich zu den Beträgen der unteren Entgeltgruppen steigen. Dadurch können Frauen in den unteren Entgeltgruppen benachteiligt werden.

Der Regelungscheck offenbart, dass in dem angewendeten Verfahren der Arbeitsbewertung Diskriminierungspotentiale vorhanden sind. In einem zweiten Schritt der Prüfung mit dem eg-check ist daher kontrolliert worden, inwieweit diese kritischen Regelungen auch eine diskriminierende Praxis bei den Eingruppierungen auf der betrieblichen Ebene bedingen. $\mathrm{Zu}$ diesem Zweck sind exemplarisch drei Paarvergleiche durchgeführt worden. Insgesamt wurden sechs Tätigkeiten mithilfe eines geschlechtsneutralen Bewertungssystems neu bewertet. Im Rahmen der Paarvergleiche wird grundsätzlich ein analytisches Verfahren verwendet, das neben den Qualifikationen und der Übernahme von Verantwortung auch psycho-soziale und physische Belastungen berücksichtigt. Zudem werden alle Kriterien und ihre jeweiligen Differenzierungsmerkmale eindeutig definiert. Ein solches Verfahren verringert den Interpretationsspielraum deutlich und bewirkt somit eine weitestgehend diskriminierungsfreie Bewertung.

Die Tätigkeiten für die exemplarischen Paarvergleiche sind systematisch nach bestimmten Kriterien ausgewählt worden. Verglichen wurden jeweils eine frauendominierte und eine männerdominierte Tätigkeit, die jeweils in derselben Entgeltgruppe eingruppiert sind. Zudem ist versucht worden, Kernstellen des Unternehmens anhand der Beschäftigtenzahl auszuwählen. Die Neubewertungen der ausgewählten Tätigkeiten wurden durch die betrieblichen AkteurInnen vorgenommen, die auch in der alltäglichen Praxis für die Eingruppierungen zuständig sind. Darunter waren sowohl Personalverantwortliche als auch Verantwortliche für den jeweiligen Fachbereich. Das detaillierte Ergebnis der Prüfung ist der nachfolgenden Tabelle zu entnehmen. 


\begin{tabular}{|c|c|c|c|c|c|c|}
\hline \multirow[b]{2}{*}{$\begin{array}{l}\text { Anforderungen im } \\
\text { Paarvergleich }\end{array}$} & \multicolumn{2}{|c|}{$\begin{array}{c}\text { Paar 1 } \\
\text { (Entgeltgruppe 4) }\end{array}$} & \multicolumn{2}{|c|}{$\begin{array}{c}\text { Paar 2 } \\
\text { (Entgeltgruppe 7) }\end{array}$} & \multicolumn{2}{|c|}{$\begin{array}{c}\text { Paar 3 } \\
\text { (Entgeltgruppe 10) }\end{array}$} \\
\hline & $\begin{array}{c}\text { Küchenwirtschafts- } \\
\text { arbeiterin }\end{array}$ & Kanalfacharbeiter & $\begin{array}{c}\text { Mitarbeiterin } \\
\text { Betriebsverwaltung }\end{array}$ & Elektriker & $\begin{array}{c}\text { Kunden- } \\
\text { korrespondentin }\end{array}$ & $\begin{array}{c}\text { Automatisierungs- } \\
\text { techniker }\end{array}$ \\
\hline $\begin{array}{l}\text { 1. Anforderungen } \\
\text { an das Wissen und } \\
\text { Können }\end{array}$ & 3 & 3 & 12 & 8 & 12 & 13 \\
\hline $\begin{array}{l}\text { 2. Anforderung an } \\
\text { psycho-soziale } \\
\text { Kompetenzen }\end{array}$ & 7 & 6 & 6 & 5 & 9 & 5 \\
\hline $\begin{array}{l}\text { 3. Anforderungen an } \\
\text { Verantwortung }\end{array}$ & 4 & 4 & 3 & 5 & 6 & 6 \\
\hline $\begin{array}{l}\text { 4. Physische } \\
\text { Anforderungen }\end{array}$ & 4 & 5 & 1 & 3 & 2 & 3 \\
\hline Gesamtsumme & 18 & 18 & 22 & 21 & 29 & 27 \\
\hline
\end{tabular}

Anmerkung: Hierbei handelt es sich um eine zusammengefasste Darstellung der Ergebnisse. Unter jedem Punkt sind noch weitere Unterpunkte zusammengefasst. Eine vollständige Darstellung der einzelnen Punkt ist dem eg-check zu entnehmen.

Wie an den Gesamtsummen zu erkennen ist, kamen die einzelnen Tätigkeiten innerhalb der Paare jeweils auf gleiche oder vergleichbare Werte. Damit kann anhand des geschlechtsneutralen Arbeitsbewertungssystems der Paarvergleiche belegt werden, dass die Eingruppierungen der ausgewählten Tätigkeiten gerechtfertig sind und an diesen Stellen keine Benachteiligungen aufgrund des Geschlechts in der betrieblichen Praxis stattfinden. Anhand der exemplarischen Prüfung kann demnach im Ergebnis belegt werden, dass in dem geprüften Unternehmen gleichwertige Arbeit von Frau- en und Männern auch mit einem gleichen Grundentgelt entlohnt wird. Eine Erklärung für dieses positive Ergebnis könnte in den geschlechtsneutralen Arbeitsbeschreibungen liegen, die zwar nicht per Tarifvertrag vorgesehen sind, aber in dem geprüften Betrieb dennoch vorgenommen werden. Abschließend hat jedoch auch eine erste kritische Sicht auf andere Entgeltbestandteile als das Grundentgelt gezeigt, dass bei den gezahlten Zuschlägen Benachteiligungen aufgrund des Geschlechts möglich sind. Hier müsste eine weitere Prüfung mit dem eg-check ansetzen.

\section{Deutscher Anwaltstag 6.-8. Juni 2013 in Düsseldorf}

\author{
Dr. Karin E. M. Kopp, LL.M. (Berkeley) \\ Regierungsrätin, München
}

Der 64. Deutsche Anwaltstag mit dem Thema „Anwaltsmarkt 2030 - Zukunft jetzt gestalten“ war ein großer Erfolg für Veranstalter und Teilnehmer. Neben Veranstaltungen, die zur Fachanwaltsfortbildung qualifizierten, brachte er auch neue Impulse für Anwältinnen. Insgesamt waren die Podien aber wieder überwiegend mit Männern besetzt.

\section{Eröffnung mit Festvortrag von Prof. Dr. Susanne Baer, Richterin des Bundesverfassungsgerichts}

Nach der Eröffnung durch den Präsidenten des Deutschen Anwaltvereins, Prof. Dr. Wolfgang Ewer und den Grußworten u.a. der Bundesministerin der Justiz, Sabine LeutheusserSchnarrenberger, wurde die Walter-Oppenhof-Medaille an DAV-Ehrenmitglied Prof. Dr. Hans-Jürgen Hellwig verliehen. Er ging in einer bewegenden Rede auf die Freiheit der Anwaltschaft und den der Individualfreiheit verpflichteten Anwalt als Interessenvertreter seiner Mandanten ein und forderte eine laufende Fortbildung, die für deutsche Anwälte bislang nicht gesetzlich vorgeschrieben sei. Nachdrücklich verlangte er nach dem Prüfungsgegenstand „Anwaltsrecht“ als Pflichtstoff in den deutschen Staatsexamina.

Anschließend hielt Frau Prof. Dr. Susanne Baer ihren sehr gelungenen Festvortrag. Sie stamme aus der weiten Juristenfamilie, wie die Anwälte, und gemeinsam gehe es darum, den Rechtsstaat zu sichern. In Ihrer kritischen Rede fragte sie u.a. nach dem Selbstbild der Anwälte und warum es noch keine pro-bono-Kultur gebe, obwohl das Rechtsdienstleistungsgesetz dies seit 1. Juli 2008 ermögliche. Auftrag des Bundesverfassungsgerichts sei eine willkürfreie Gleichstellung in allen Lebensbereichen. Nachdem sich der Gesetzgeber seit 2001 auch zur Gleichstellung gleichgeschlechtlicher Lebenspartnerschaften verpflichtet habe, sei die heute (am 6. Juni 2013) veröffentlichte Entscheidung zur Erstreckung des Ehegattensplittings auf eingetragene Lebenspartnerschaften folgerichtig. Historisch ging sie auf die Gleichschaltungsgesetze von 1933 ein und erinnerte daran, dass auch die Anwälte an der Exklusion ihrer jüdischen Konkurrenz interessiert waren. ${ }^{1}$ 